\title{
KULEUVEN
}

\begin{tabular}{|c|c|}
\hline Citation & $\begin{array}{l}\text { Dhaini, S. R., Zúñiga, F. (2015) } \\
\text { Absenteeism and Presenteeism among Care Workers in Swiss Nursing } \\
\text { Homes and Their Association with Psychosocial Work Environment: A } \\
\text { Multi-Site Cross-Sectional Study } \\
\text { Gerontology, Epub ahead of print. }\end{array}$ \\
\hline Archived version & $\begin{array}{l}\text { Post-print. Author manuscript: the content is virtually identical to the content } \\
\text { of the published paper, but without the final typesetting by the publisher }\end{array}$ \\
\hline Published version & \\
\hline Journal homepage & http://www.karger.com/Journal/Home/224091 \\
\hline Author contact & $\begin{array}{l}\text { Sabina.degeest@med.kuleuven.be } \\
\text { Sabina.degeest@unibas.ch } \\
+32(0) 16373294 \\
+41(0) 612670916\end{array}$ \\
\hline IR & https://lirias.kuleuven.be/cv?u=U0005386 \\
\hline
\end{tabular}

(article begins on next page) 
Manuscript submission to the Journal of Gerontology

\section{"Absenteeism and Presenteeism among Care Workers in Swiss Nursing Homes and their Association with Psychosocial Work Environment: a Multi-site Cross-sectional Study"}

Running title: Absenteeism and Presenteeism in Swiss Nursing Homes

Suzanne R. Dhaini ${ }^{1}$ MA, Franziska Zúñiga ${ }^{1}$ MSc, Dietmar Ausserhofer ${ }^{1,2}$ PhD, Michael Simon ${ }^{1,3}$ $\mathrm{PhD}$, Regina Kunz ${ }^{4} \mathrm{MD}$, Sabina De Geest ${ }^{1} \mathrm{PhD}$, Rene Schwendimann ${ }^{1} \mathrm{PhD}$

${ }^{1}$ Institute of Nursing Science, University of Basel, Bernoullistrasse. 28, 4056 Basel, Switzerland Tel. +41(0)612670919

Fax +41(0)612670955

Suzanne.dhaini@unibas.ch

Franziska.zuniga@unibas.ch

Dietmar.ausserhofer@unibas.ch

Michael.simon@unibas.ch

Sabina.degeest@unibas.ch

$\underline{\text { Rene.schwendimann@unibas.ch }}$

${ }^{2}$ Claudiana, University of Applied Science, Lorenz-Böhler-Str. 13, I-39100 Bozen - Bolzano (BZ), Italy

Tel. $+39-0471-067290$

Fax. +39-0471-067240

dietmar.ausserhofer@claudiana.bz.it

${ }^{3}$ University Hospital Inselspital Bern, Nursing \& Midwifery Research Unit, 3010 Bern, Switzerland

${ }^{4}$ Swiss Academy of Insurance Medicine, University Hospital Basel, Petersgraben 4, 4031, Basel, Switzerland

Tel.+41 612655568

Fax +4161265 5534

regina.kunz@usb.ch

\section{Correspondence to}

René Schwenndimann, $\mathrm{PhD}$

Institute of Nursing Science,

University of Basel,

Bernoullistrasse 28,

4056-Basel, Switzerland

Tel: (+41) 612670919

Fax: (+41) 612870955

rene .schwendimann@unibas.ch

\section{ABSTRACT}

Background: Worker productivity is central to the success of an organization such as health 
care institutions. However, both absenteeism and presenteeism impair that productivity. While various hospital studies have examined the prevalence of presenteeism and absenteeism and its associated factors among care workers, evidence from nursing home settings is scarce.

Objective: To explore care workers' self-reported absenteeism and presenteeism in relation to nursing homes' psychosocial work environment factors.

Methods: A cross-sectional study utilized survey data of 3,176 professional care workers in 162 Swiss nursing homes collected between May 2012 and April 2013. A GEE ordinal logistic regression model was used to explore associations between psychosocial work environment factors (leadership, staffing resources, work stressors, affective organizational commitment, collaboration with colleagues and supervisor, support from other personnel, job satisfaction, job autonomy) and self-reported absenteeism and presenteeism.

Results: Absenteeism and presenteeism were observed in $15.6 \%$ and $32.9 \%$ of care workers, respectively. While absenteeism showed no relationship with the work environment, low presenteeism correlated with high leadership ratings (OR 1.22, CI 1.01-1.48) and adequate staffing resources (OR 1.18, CI 1.02-1.38).

Conclusion: Self-reported presenteeism is more common than absenteeism in Swiss nursing homes, and leadership and staffing resource adequacy are significantly associated with presenteeism, but not with absenteeism.

Keywords absenteeism, care worker, nursing home, presenteeism, work environment

\section{INTRODUCTION}

Worker productivity is central to the success of any organization [1]. However, both absenteeism, i.e., missing shifts because of feeling unwell or unfit to work, and presenteeism, i.e., working despite feeling unwell or unfit to work, impair that productivity. With absenteeism, as employees contribute nothing to the organization's operation, productivity 
loss per absent employee is $100 \%$ [2]. Presenteeism is considered the opposite of absenteeism [3] but it decreases productivity making illness at work a costly affair [1,4]. The two concepts are closely linked: frequent presenteeism is associated with subsequent longterm absenteeism [5].

The concept of presenteeism first appeared in empirical literature in the 1990s, [6] when employers noticed that not only absenteeism but also presenteeism drains productivity [7]. Since then, studies on the general population have indicated that both absenteeism and presenteeism are strong predictors of future poor health, physical complaints, low mental well-being, and low work ability [8].

In healthcare, previous studies have shown that high rates of presenteeism are common among nursing care workers, regardless of their work setting [9-11]. For example, in $2011,49 \%$ of the Swedish public health sector workers (including hospitals and primary care workers) reported frequent presenteeism in the preceding year [12].

Research $[9,11,13]$ has suggested that the ability to work through illness depends on work demands, workload, and perceived job stress. Hence, if the ill person perceives that coworkers will not be able to compensate for their absence, they commonly work despite illness [9, 13]. For example, care workers' daily responsibilities involve providing service and responding to patients' needs. If the ill persons perceive that the care workers present will not be able to compensate for their absence, they commonly work despite illness $[9,14]$. In nursing homes, residents who can no longer reliably perform the basic activities of daily living in their homes require $24 / 7$ direct care. As a result, nursing home care workers need to perform many physically and emotionally straining activities that risk compromising their health [15].

Several studies on the general population have indicated relationships between absenteeism and presenteeism $[13,16]$. Workers who reported calling in sick also tended to 
report working while ill [13]. Individual characteristics such as occupation and gender [10], and work related factors including a strong commitment to work [13] were found to influence both absenteeism and presenteeism [17]. Recent studies have linked negative perceptions of the work environment [18] -e.g., poor collaboration with colleagues [19] and time pressure $[13,20]$ with presenteeism. In a Scandinavian study on the care of older people, researchers showed that high presenteeism was associated with high workloads and elevated time pressure [11].

Compared to absenteeism, presenteeism has been relatively less researched, probably because it is harder to track associated cost [21]. Nonetheless, existing studies have highlighted the magnitude of presenteeism not only by its cost of lost productivity, but also by negatively affecting quality patient care [21]. While various hospital studies in Europe and the U.S. have examined the prevalence of presenteeism (ranging from $21.9 \%$ to $62 \%$ ), and its associating factors $[14,19,21]$, evidence from nursing home settings is scarce. Although the relationships between absenteeism and presenteeism are unclear, Kristensen argued convincingly that both behaviours are outcomes of the same decision process [22]. Therefore, examining risk factors for absenteeism in nursing home care workers' psychosocial work environments (e.g., leadership, collaboration with supervisor, work stressors, staffing resources) could improve our perception of presenteeism [13]. This study adds to the body of knowledge on absenteeism, and to the growing literature on presenteesim in healthcare.

\section{THEORETICAL BACKGROUND}

The WHO Healthy Workplace Model (figure 1) [1] and its "Business Case" framework (figure 2) [1] contribute to the understanding of the work environment's relationships with absenteeism and presenteeism. All workplaces require healthy workers to sustain the organization [1]. Therefore, the WHO model ties unhealthy and unsafe workplaces to work- 
related physical and mental illnesses, very likely increasing the risks of both absenteeism and presenteeism. The WHO's key components of a healthy workplace correspond to four domains: 1) the physical work environment (e.g. chemical hazards and biological hazards); 2) the psychosocial work environment (e. g organization daily practices and workplace stressors); 3) personal health resources (e.g. physical inactivity from long working hours, poor diet due to lack of time); and 4) enterprise community involvement (e.g. supporting community screening and treatment, providing leadership and expertise related to workplace health and safety to other organizations). Using data from the Swiss Nursing Homes Human Resources Project (SHURP), we explored psychosocial work environment factors' associations with absenteeism and presenteeism in nursing home care workers. Rooted in organizational culture and daily practice, these factors can include, among others, work stressors, staffing resource inadequacy, poor leadership, poor co-worker support, poor collaboration with management or among colleagues, low job autonomy, low job satisfaction, and poor affective organizational commitment [1]. The variables are defined by the WHO model but operationalized to meet the study purposes.

Exploring absenteeism and presenteeism in nursing homes serves two important purposes. First, determining the prevalence of each provides insight into their magnitude as nursing workforce outcomes in long-term care settings. Second, as work environment factors can influence employee productivity-via absenteeism and presenteeism-they also influence an organization's sustainability [1]. Accordingly, this study had two aims: 1) to determine the prevalence of absenteeism and presenteeism among professional care workers in Swiss nursing homes; and 2) to explore psychosocial work environment factors' associations with absenteeism and presenteeism. 


\section{METHODS}

\section{Study design, setting, and sample}

This is a secondary data analysis of the multi-center, cross-sectional SHURP study, which included a random sample of 162 nursing homes across Switzerland, stratified according to language region and size. Nursing homes smaller than 20 beds, residential care homes, and rehabilitation clinics for geriatric patients were excluded. Full details of the sampling and survey methods used are provided elsewhere [23].

In the parent study, 6,947 questionnaires were distributed to care workers, of which $5,323(76.6 \%)$ were returned. Care workers of all educational levels (registered nurses, licenced practical nurses, certified nursing assistants, and nurse aides) who provided direct care to the nursing home residents were invited to complete the questionnaire survey. Care workers who worked fewer than 8 hours weekly, had been employed less than 1 month on the unit, or were students were excluded from the parent study. In the current study, only care workers without leadership positions were included, leading to a sub-sample of 3,176 professional care workers.

\section{Data sources, variables and measurements}

Socio-demographic and professional data on care workers, including their perceptions of their work environment, work stressors, health status, absenteeism, and presenteeism, were collected using the SHURP study's Care Worker Personnel Questionnaire. Nursing home facility characteristics were captured from the SHURP Facility Profile.

The SHURP study team established the content validity of each scale used, testing the relevance of each variable and scale separately and adjusting them as necessary until all achieved desirable item content validity index (I-CVI) or scale content validity index (S- 
CVI) ratings. All items of the care worker questionnaire were translated into German, French, and Italian. Items were verified with the original language version by comparison of its back translation. Then, they were tested for relevance with gerontological experts in the field to check content validity, and pre-tested for their comprehensibility with end-user focus group. Further information related to the development of the questionnaire and the survey validity pre-testing are described elsewhere [23].

\section{Variables and measurements}

The current study used the following dependent, independent and control variables.

\section{Dependent Variables}

\section{Absenteeism}

Absenteeism was measured via an investigator-developed item measuring how many days (if any) in the previous 4 weeks care workers had not attended work due to feeling ill and unfit for work. Respondents answered by number of days. Numbers were later grouped into three categories ( $0=0$ days, $1=1-2$ days, $2=3$ or more days $)$ as in presenteeism [10].

\section{Presenteeism}

Presenteeism was measured via an investigator-developed item measuring how many days (if any) in the previous 4 weeks care workers had attended work in spite of feeling ill and unfit for work. Respondents answered by number of days. Answers were later grouped into three categories $(0=0$ days, $1=1-2$ days, $2=3$ or more days)[10].

\section{Independent Variables}

\section{Psychosocial work environment risk factors}

Care workers' perceptions of their nursing homes' leadership and staffing adequacy were measured via items from two subscales of the Practice Environment Scale of the Nursing Work Index (PES-NWI) questionnaire: "Nurse manager ability, leadership, and 
support of care workers" (Cronbach alpha 0.84) and "Staffing and resources adequacy" (Cronbach alpha 0.74)[24]. These were adapted for nursing home use with 4-point Likerttype scale ( $1=$ strongly disagree, $2=$ rather disagree, $3=$ rather agree, $4=$ strongly agree). The leadership items asked about the extent to which respondents perceived their unit supervisors as supportive and competent leaders, mistakes were used as learning opportunities, care workers were rewarded or otherwise recognized for work well done, and the unit leaders supported them in decision making. Items on staffing adequacy asked about perception of enough staff on duty to complete all necessary work, to provide quality care, and to discuss resident problems.

Work stressor items were selected from the Health Professions Stress Inventory (HPSI) $[25,26]$ to measure the frequency of several work-related stressors. These were measured via a 5 -point Likert-type scale $(0=$ never, $1=$ seldom, $2=$ sometimes, $3=$ often, $4=$ very often). The instrument was reduced from 30 to 12 items in order to reduce the SHURP`s survey burden (time spent filling out questionnaires). The reduction was based on the ratings of experts from the gerontological field (holding at least a Certificate of Advanced Studies up to a Master's degree with experience in nursing home care) with regards to the relevance of each question. The SHURP team asked the experts to rate each item for its understandability for nursing home personnel (yes/no), and for its relevance concerning resident safety on a 4-point scale (1=not relevant, $2=$ somewhat relevant, $3=$ quite relevant, $4=$ very relevant). The item content validity index (I-CVI) was calculated for each item as percentage of experts who rated it 3 or 4 . The average scale content validity (S-CVI/Ave) was calculated as the mean of all I-CVI. The SHURP group's psychometric analysis of the remaining 12 items produced 3 sub-scales tested for internal consistency (Cronbach`s alpha) and measuring stress-producing factors: (1) workload (Cronbach's alpha: 0.73), (2) lack of job preparation (Cronbach's alpha 0.63), and (3) conflict and lack of recognition 
(Cronbach's alpha 0.76). Stress due to workload was measured via three items on dealing with difficult situations, having too much work to do, and being understaffed. The three items measuring stress due to lack of job preparation asked about fear of making mistakes, being overwhelmed when caring for terminally ill residents, and not being prepared to meet the residents' needs. Regarding conflict and lack of recognition, six items asked about disagreements with other professionals, conflicts with superiors, lack of information, not being asked about one's opinion, being underpaid, and underuse of skills.

Affective organizational commitment was adopted from the "Questionnaire for the Assessment of Affective, Costing, and Normative Commitment to the Organization, the Profession/Activity and Employment Form" (COBB)[27], using five items from the Affective Commitment sub-scale (Cronbach`s alpha 0.86), and rated on a 5-point Likerttype scale ( $1=$ strongly disagree, $2=$ slightly disagree, $3=$ neutral, $4=$ slightly agree, $5=$ strongly agree). These items assessed respondents' feelings about the organizations employing them, including how happy they would be to spend the next years with their current organization, the strength of their sense of belonging to that organization, their level of emotional attachment to their organization, and how well their personal ideals fit with those of the organization.

Items on collaboration with colleagues and with unit supervisors were adopted from the Safety Attitude Questionnaire (SAQ)[28]. On 4-point Likert-type scales, respondents rated the quality of each level of collaboration $(1=$ very low, $2=$ rather low, $3=$ rather high, 4=very high). A “don’t know” option was also provided (treated as missing in the analysis). For conformity with the study's data on risk factors, answers were dichotomized $(0=$ very low, rather low; $1=$ rather high, very high). One item on support from other personnel to care for residents was also selected from the SAQ and rated on a 5-point Likert-type scale (1=strongly disagree, $2=$ =slightly disagree, $3=$ neutral, $4=$ slightly agree, $5=$ strongly agree). 
This also included the "don't know" answer option. As above, answers were dichotomized for data conformity $(0=$ strongly disagree, slightly disagree, neutral; $1=$ slightly agree, strongly agree).

To measure autonomy at work, a single investigator-developed item asked care workers to rate the extent to which they decided independently how to perform their work. This item was rated on a 4-point Likert-type scale (1=strongly disagree, $2=$ slightly disagree, $3=$ slightly agree, 4=strongly agree). Again, responses were dichotomized ( $0=$ strongly disagree, slightly disagree; $1=$ slightly agree, strongly agree). Job satisfaction was measured via another investigator-developed item. On a 4-point Likert-type scale (1=very dissatisfied, $2=$ rather dissatisfied, $3=$ rather satisfied, $4=$ very satisfied), this assessed each care worker's overall satisfaction with his/her current job in the nursing home. As above, answers were dichotomized as positive or negative ( $0=$ very dissatisfied, rather dissatisfied; $1=$ rather satisfied, very satisfied).

\section{Control Variables}

\section{Facility characteristics}

Facility characteristics included size (small: 20-49 beds; medium: 50-99 beds; or large: $\geq 100$ beds), language region (German-, French-, or Italian-speaking area), and ownership status (private, private subsidized, public).

\section{Care worker socio-demographic and professional characteristics}

Care worker socio-demographic data were collected on age (date of birth), gender, educational level (i.e., registered nurse, licensed practical nurse, certified nursing assistant, nurses aide), professional experience in nursing in years (number of years in nursing), percentage of full-time employment (corresponding to number of hours worked per week, ranging from $20 \%$ ( $8 \mathrm{hrs} /$ week) to $100 \%$ (42hrs/week)), agency staff (i.e., a temporary (vs. permanent) position), usual work shifts (days, evenings, nights, or regularly rotating shifts), 
and frequency of overtime (less frequently, every 5-7 working days, every 2-4 working days, almost every shift). Age (up to 30 years; $31-40 ; 41-50 ;>50$ years) and professional experience in nursing (up to 5 years; 6-10 years; 11-15 years; 16-20 years; >20 years) were then categorized for analysis purposes. Professional categories were based on 5 nursing education levels: registered nurses (three to six years of education, leading to a diploma in nursing, bachelor's degree (BSc.N. or equivalent) or higher); licensed practical nurses (LPN) (three years of education); certified nursing assistants (CNA) (two years of education); and nurse aides (short courses or on-the-job training).

\section{Care worker health status}

Care workers' physical health status was assessed using a health index designed to minimize the number of health-related outcome variables. Five items were selected from the Swiss Health Survey [29] to gather self-reported data on back pain, joint pain, tiredness, sleeplessness, and headache during the preceding 4 weeks, with each measured on a 3-point Likert-type scale ( $1=$ not at all, $2=$ a little bit, $3=$ strongly). The index score was calculated as sum of item scores (range: 5-15) over number of items $(n=5)$ minus 5 (allowing the index to start with 0 for "no health complaints"). Higher index scores (max: 10) signify more health problems. This index is based on principal component analysis of the 5 items, with one factor explaining $45 \%$ of the variance. Item loadings ranged between 0.62 and 0.74 (Cronbach`s alpha 0.69).

The care worker`s mental health status-emotional exhaustion-was measured on a 7point Likert-type scale (ranging from $0=$ never, to $6=$ daily) using the item "feeling exhausted from work" from the Maslach Burnout Inventory (MBI)[30]. The validity of measuring emotional exhaustion with a single item is described elsewhere. ${ }^{[31]}$ 


\section{Data collection and Analysis}

The SHURP survey was administered between May 2012 and April 2013. Detailed information on data collection is provided elsewhere [23].

As facility and care worker characteristics, including health status, have been extensively investigated in previous studies, showing positive relationships with absenteeism and presenteeism, they were used here as control variables[19, 32]. To address aim 1, we calculated descriptive statistics (frequencies, percentages, means, and standard deviations). For aim 2, we first analyzed the univariate associations between facility and care worker characteristics (including health status) and absenteeism and presenteeism. We used generalized estimation equation (GEE) multiple regression models to account for the clustering of care workers in nursing home units. Next, adjusting for facility characteristics and care worker characteristics (including health status), we used ordinal logistic GEE regression to estimate odds ratios (ORs) and 95\% confidence intervals (CIs) for psychosocial work environment risk factors. We also assessed multi-collinearity of all work environment factors with variance inflation factor (VIF). Based on this VIF with all values remaining below the threshold of 5, all variables were included in the analysis[33]. Missing values analysis showed less than $5 \%$ of responses missing per variable, with approximately $23 \%$ of respondents $(\mathrm{n}=938)$ omitting one or more responses. To explore any pattern of missed data, we analysed the sensitivity of the entire sample $(n=4,014)$ against that of the subgroup who submitted complete response sets $(n=3,176)$. To compare means of each variable examined in this study, we calculated Cohen's d. Calculated differences were small (Cohen's $\mathrm{d}<0.2$ )[34], with similar inferences. All data analyses were conducted using IBM/SPSS for Mac Statistics 21.0. We report only adjusted results of our analysis. 


\section{Ethical approval}

All participating nursing home administrators and nursing directors gave written informed consent to participate in the SHURP study. Care workers' voluntary and confidential return of their SHURP questionnaires was treated as informed consent. This study was covered by the Swiss nursing homes human resources Project (SHURP), for which the ethic committee of the state of 'beider Basel' (Ref.Nr EK:02/12) granted approval.

\section{RESULTS}

\section{Description of sample}

Overall, this study included data supplied by 3'176 care workers in 162 nursing homes. Slightly fewer than half of participating nursing homes were medium in size (46.3\%); one third had public ownership (37\%). Three-quarters $(75.9 \%)$ were located in Switzerland's German-speaking area. Table 1 summarizes the participants' characteristics and psychosocial work environment factors.

Across all facilities, a large majority (92.2\%) of care workers were female; fewer than a third were registered nurses (27.9\%). Roughly a third (32.7\%) were 50 years of age or older and roughly a quarter $(24.6 \%)$ had 21 or more years of nursing experience. The majority $(75.3 \%)$ were employed more than $50 \%$ and not working for an agency $(93.7 \%)$. Fewer than half $(44.7 \%)$ reported working mostly day shifts. Slightly more than a third (37.7\%) reported incidences of work-related emotional exhaustion ranging from several times a month to daily. Overall, respondents reported positive psychosocial work environments, with high levels of collaboration both among colleagues (96.0\%) and with unit supervisors (90.6\%), strong levels of support from other personnel (88.8\%), autonomy at work $(81.1 \%)$, and job satisfaction $(87.5 \%)$. 


\section{Prevalence of absenteeism and presenteeism among care workers}

Of the 3,176 care workers who submitted eligible questionnaires, $14.6 \%$ reported absenteeism, with $32.9 \%$ reporting presenteeism for at least one shift during the month prior to the survey (Table 2); $5.6 \%$ reported three or more days of absenteeism; and $16.8 \%$ reported three or more days of presenteeism. Conversely, $85.4 \%$ and $67 \%$ of all participants respectively reported zero days of either absenteeism or presenteeism.

\section{Associations of psychosocial work environment factors with absenteeism and presenteeism}

Absenteeism showed no significant association with any psychosocial work environment factor investigated in this study. However, presenteeism was associated with two psychosocial work environment risk factors (Table 3): perceptions of supportive leadership (OR 1.22, CI 1.01-1.48) and adequate staffing resources (OR 1.18, CI 1.02-1.38) both increased the odds of low presenteeism. No other associations with psychosocial work environment factors were statistically significant.

\section{DISCUSSION}

While this study found no significant associations between psychosocial work environment risk factors and self-reported absenteeism, analyses indicated that both perception of supportive leadership and staffing resource adequacy correlated with lower self-reported presenteeism. While our findings on absenteeism do not support previous research, our measured 37\% prevalence of self-reported presenteeism [10] is congruent with earlier observations $[10,14]$.

Overall, nursing home care workers' self-reported presenteeism in the month prior to the survey was more common than similarly-reported absenteeism over the same period. While the prevalence of self-reported absenteeism of three and more days was fairly low (5.6\%), it was slightly higher than that self-reported for US healthcare workers in the same 
year (2012) (4.5\%)[35]. Unfortunately, the US findings provided no nursing homespecific figures. Also, US healthcare workers may not enjoy the same protections as in the Switzerland, where missing a shift may entail losing a day`s pay.

Comparing various occupations of the general population in Sweden (e.g., care providers and school teachers), Aronsson et al. (2000) observed higher presenteeism among female healthcare workers compared with female workers in other occupations [10]. This supports Szymczak, J.E., et al.'s (2015) conclusion that the nature of a caring relationship between the care worker and the patient decreases the likelihood of absenteeism and magnifies the tendency to work while ill [14], and John's (2010) postulation that the work identity of the care worker is linked to helping the vulnerable patient [36]. Recent findings in one US hospital suggested that care workers were ambivalent both about which symptoms and illnesses constituted being too sick to work, and about whether their organizations' sickness relief systems were adequate [14].

As noted above, in contrast to previous studies on predictors of absenteeism in nursing homes [37, 38], we found no association between psychosocial work environment and self-reported absenteeism. While the perception of a supportive leadership, supportive peer relationships [37, 38], appropriate job training, job satisfaction [38], and affective organizational commitment[39] have all been linked to reduced rates of absenteeism in other European healthcare settings, this study confirmed no such relationships. However, in accordance with one study [20], we found that job satisfaction did not influence the probability of absenteeism. A plausible explanation for inconsistent study findings would be the broad range of workplace cultures, social, legal, and economic contexts involved. Varying from one country or culture to another, all these factors impact the traditions and practices of healthcare workers, potentially influencing their attitudes towards absenteeism [10]. 
Our findings suggest that absenteeism cannot be fully explained by care workers' work attitudes [27, 40]. For example, personal factors such as health status have been found to predict the probability of absenteeism [20] and influence the relationship between affective organizational commitment and absenteeism [27].

One novel finding was that an increase in the perception of a supportive leadership and adequate staffing resources ratings increased the odds of self-reported low presenteeism. This is very possibly because care workers confident that their perception of a supportive leadership and/or the available staffing resources are adequate to counterbalance absences are more comfortable about staying home while ill. Our findings corroborate those of a previous study on the general Danish workforce [13], indicating that work-related factors, e.g., high levels of time pressure and poor social support, were predictors of presenteeism. In a much more recent study [39] using a univariate model, affective organizational commitment was inversely related to presenteeism, which was confirmed in our simple regression model (not shown). In our multivariate model, affective organizational commitment lost its significance in combination with all other variables. As no previous studies have specifically examined presenteeism in relation to care workers` perception of a supportive leadership and staffing resource adequacy, these findings warrant further investigation.

Finally, our findings suggest that, as psychosocial work environment factors, the perception of a supportive leadership and staffing resource adequacy are important in predicting presenteeism but not absenteeism. Compared to absenteeism, there is no golden rule to describe whether presenteeism is a desired or undesired behaviour in health care. In our opinion, showing up to work while ill could be a sign of commitment as discussed earlier, and fear of loosing one`s job when being absent too often. Nevertheless, one could 
also see presenteeism as a risk of poor performance due to illness, as a sign of lost productivity [21].

\section{Strengths and limitations}

The SHURP study is the first comprehensive national survey health of care workers in Swiss nursing homes to gather data both on work environment factors and on absenteeism and presenteeism. The findings of this secondary analysis, however, should be interpreted in light of certain limitations. First, the definition of illness and "staffing adequacy" used in this study, relied solely on the respondents' subjective perceptions of their health, and staffing level, with no independent evaluation of their objective health status and "adequacy" standards in staffing. Second, the cross-sectional design does not allow causal inferences about the observed relationships between variables. Nevertheless, our findings will inform stakeholders and future interventional studies about system factors associated with care workers' presenteeism at the levels of the organization and the individual care worker. Third, quantifying presenteeism relied solely on self-report measures. Fourth, the secondary data analysis limited our ability to fully evaluate the impacts of all of the proposed model's domains (Fig.1) on care workers' health.

\section{CONCLUSIONS}

This is the first study in a representative sample of Swiss nursing homes to examine self-reported absenteeism and presenteeism among professional care workers in relation to selected psychosocial work environment factors. Our findings indicate that self-reported presenteeism is more common than absenteeism in Swiss nursing homes, and that the perception of a positive leadership and staffing resource adequacy are significant associations with presenteeism, but not absenteeism. Care workers` presenteeism in nursing homes is an area that has been overlooked. Focusing on presenteeism is reasonable for nurse directors and administrators who want to promote nurses` health in order to sustain the 
organization. Future analysis is required to assess how presenteeism might influence quality of care. Additional analysis is needed, taking into account the four work environment domains of the proposed WHO workplace model.

Fund: This study was funded by the Swiss Health Observatory, Nursing Science Foundation Switzerland, University of Basel's Research fund 2012, Swiss Alzheimer Association, and an anonymous sponsor.

Conflict of interest: No conflicts of interest declared.

Acknowledgement: Special thank goes to the nursing homes and care workers for participating in the SHURP study, and to Chris Shultis for editing our manuscript.

\section{REFERENCES}

1. Burton J. WHO health workplace framework and model: background and supporting literature and practice. Geneva, Switzerland: WHO Headquarters, 2010 February. Report No. 2. Bockerman P, Laukkanen E. Predictors of sickness absence and presenteeism: does the pattern differ by a respondent's health? JOEM/ American College of Occupational and Environmental Medicine. 2010;52(3):332-5. Doi: 10.1097/JOM.0b013e3181d2422f. 3. Schultz AB, Edington DW. Employee health and presenteeism: a systematic review. J Occup Rehabil. 2007;17(3):547-79. Doi: 10.1007/s10926-007-9096-x. 
4. Hemp P. Presenteeism: at work--but out of it. Harvard Business Review. 2004;82(10):49-58, 155. Doi: 10.1080/02678373.2014.909904.

5. Hansen CD, Andersen JH. Sick at work--a risk factor for long-term sickness absence at a later date? JECH. 2009;63(5):397-402. Doi: 10.1136/jech.2008.078238.

6. Bockerman P, Laukkanen E. What makes you work while you are sick? Evidence from a survey of workers. Eur J Publich Health. 2010;20(1):43-6. Doi: 10.1093/eurpub/ckp076.

7. Demerouti E, Le Blanc MP. Present but sick: a three-wave study on job demands, presenteeism and burnout. Career Development International. 2009;14(1):50-68. Doi.

8. Gustafsson K, Marklund S. Consequences of sickness presence and sickness absence on health and work ability: a Swedish prospective cohort study. International JOMEM. 2011;24(2):153-65. Doi: 10.2478/s13382-011-0013-3.

9. Aronsson G, Gustafsson K. Sickness presenteeism: prevalence, attendance-pressure factors, and an outline of a model for research. JOEM / American College of Occupational and Environmental Medicine. 2005;47(9):958-66. Doi.

10. Aronsson G, Gustafsson K, Dallner M. Sick but yet at work. An empirical study of sickness presenteeism. JECH. 2000;54(7):502-9. Doi: 10.1136/jech.54.7.502.

11. Elstad JI, Vabo M. Job stress, sickness absence and sickness presenteeism in Nordic elderly care. SCAND J PUBLIC HEALT. 2008;36(5):467-74. Doi: $10.1177 / 1403494808089557$.

12. Dellve L, Hadzibajramovic E, Ahlborg G, Jr. Work attendance among healthcare workers: prevalence, incentives, and long-term consequences for health and performance. $\mathrm{J}$ Adv Nurs. 2011;67(9):1918-29. Doi: 10.1111/j.1365-2648.2011.05630.x.

13. Hansen CD, Anderson JH. Going ill to work : what personal circumstances, attitudes and work-related factors are associated with sickness presenteeism? Soc Sci Med. 2008;67:956-64. Doi: 10.1016/j.socscimed.2008.05.022.

14. Szymczak JE, Smathers S, Hoegg C, Klieger S, Coffin SE, Sammons JS. Reasons Why Physicians and Advanced Practice Clinicians Work While Sick: A Mixed-Methods Analysis. JAMA pediatrics. 2015. Doi: 10.1001/jamapediatrics.2015.0684.

15. Dhaini S, Zúñiga F, Ausserhofer D, Simon M, Kunz R, De Geest S, et al. Care workers health in swiss nursing homes and its association with psychosical work environment: a cross-sectional study. IJNS. in press. Doi:

http://dx.doi.org/10.1016/j.ijnurstu.2015.08.011

16. Leineweber C, Westerlund H, Hagberg J, Svedberg P, Alexanderson K. Sickness presenteeism is more than an alternative to sickness absence: results from the populationbased SLOSH study. Int Arch Occup Environ Health. 2012;85(8):905-14. Doi: 10.1007/s00420-012-0735-y.

17. Bockerman P, Ilmakunnas P. Interaction of working conditions, job satisfaction, and sickness absences: evidence from a representative sample of employees. Soc Sci Med (1982). 2008;67(4):520-8. Doi: 10.1016/j.socscimed.2008.04.008.

18. Pilette PC. Presenteeism in nursing: a clear and present danger to productivity. JONA. 2005;35(6):300-3. Doi.

19. d'Errico A, Viotti S, Baratti A, Mottura B, Barocelli AP, Tagna M, et al. Low back pain and associated presenteeism among hospital nursing staff. J Occup Health. 2013;55(4):276-83. Doi.

20. Rantanen I, Tuominen R. Relative magnitude of presenteeism and absenteeism and work-related factors affecting them among health care professionals. Int Arch Occup Environ Health. 2011;84(2):225-30. Doi: 10.1007/s00420-010-0604-5. 
21. Letvak SA, Ruhm CJ, Gupta SN. Nurses' presenteeism and its effects on selfreported quality of care and costs. Am J Nurs. 2012;112(2):30-8; quiz 48, 39. Doi: 10.1097/01.NAJ.0000411176.15696.f9.

22. Kristensen T. Sickness absence and work strain among Danish slaughterhouse workers: an analysis of absence from work regarded as coping behaviour. Soc Sci Med. 1991;32:15-27. Doi.

23. Schwendimann R, Zuniga F, Ausserhofer D, Schubert M, Engberg S, De Geest S. Swiss Nursing Homes Human Resources Project (SHURP) protocol of an observational study. J Adv Nurs. 2013;70(4):915-26. Doi: 10.1111/jan.12253.

24. Lake ET. The nursing practice environment: measurement and evidence. Med Care Res Rev. 2007;64(2(suppl)):104S-22S. Doi: 10.1177/1077558707299253.

25. Akhtar S, Lee, J.S. Confirmatory factor analysis and job burnout correlates of the Health Professions Stress Inventory. Psychological Reports 2002;90(1):243-50. Doi.

26. Wolfgang AP. The Health Professions Stress Inventory. Psychological Reports. 1988;62(1):220-2. Doi: 10.2466/pr0.1988.62.1.220.

27. Felfe J, Six B, Schmoock R, Knorz C. Questionnaire for the assessment of affective, calculative and normative commitment to the organization, the profession / activity and employment form (COBB). In Glöckner-Rist, A. (Eds), compilation of social science items and scales. CIS Version 14:00. Bonn:GESIS2010.

28. Sexton JB, Helmreich RL, Neilands TB, Rowan K, Vella K, Boyden J, et al. The Safety Attitude Questionnaire: psychometric properties, benchmarking data, and emerging research. BMC Healh Services Reasearch. 2006;6(44). Doi: 10.1186/1472-6963-6-44.

29. OFS. Swiss Health Survey 2012. Switzerland: Federal Department of Home Affairs, Health OFoSDoS; 2012.

30. Maslach CaJ, S.E. The Measurement of Experienced Burnout. Journal of Occupational Behaviour. 1981;2(2):99-113. Doi.

31. West CP, Dyrbye LN, Sloan JA, Shanafelt TD. Single item measures of emotional exhaustion and depersonalization are useful for assessing burnout in medical professionals. $\mathrm{j}$ Gen Intern Med. 2009;24(12):1318-21. Doi: 10.1007/s11606-009-1129-z.

32. Roelen CA, Bultmann U, Groothoff J, van Rhenen W, Mageroy N, Moen BE, et al. Physical and mental fatigue as predictors of sickness absence among Norwegian nurses. Res Nurs Health. 2013;36(5):453-65. Doi: 10.1002/nur.21558.

33. Field A. Discovering Statistics Using SPSS. Third Edition ed. London: SAGE; 2009.

34. Enders KE. Applied missing data analysis. 72 Spring Street, New York, NY 10012: The Guilford Press; 2010.

35. BLS. Labor force statistics from the current population survey. Washington, DC: United States Department of Labor, 2012.

36. John G. Presenteeism in the workplace: A review and research agenda. Journal of Organizational Behavior. 2010;31:519-42. Doi: 10.1002/job.630.

37. Clausen T, Nielsen K, Carneiro IG, Borg V. Job demands, job resources and longterm sickness absence in the Danish eldercare services: a prospective analysis of registerbased outcomes. J Adv Nurs. 2012;68(1):127-36. Doi: 10.1111/j.1365-2648.2011.05724.x. 38. Sanders K, Nauta A. Social cohesiveness and absenteeism: The relationship between characteristics of employees and short-term absenteeism within an organization. Small Group Research. 2004;35(6):724-41. Doi: 10.1177/1046496404267186.

39. Graf E, Cignacco E, Zimmermann K, Zúñiga F. Affective Organizational Commitment in Swiss Nursing Homes: A Cross-Sectional Study. The Gerontologist. 2015;00(00):1-15. Doi: 0.1093/geront/gnv053. 
40. Davey MM, Cummings G, Newburn-Cook CV, Lo EA. Predictors of nurse absenteeism in hospitals: a systematic review. J Nurs Manag. 2009;17(3):312-30. Doi: 10.1111/j.1365-2834.2008.00958.x. 


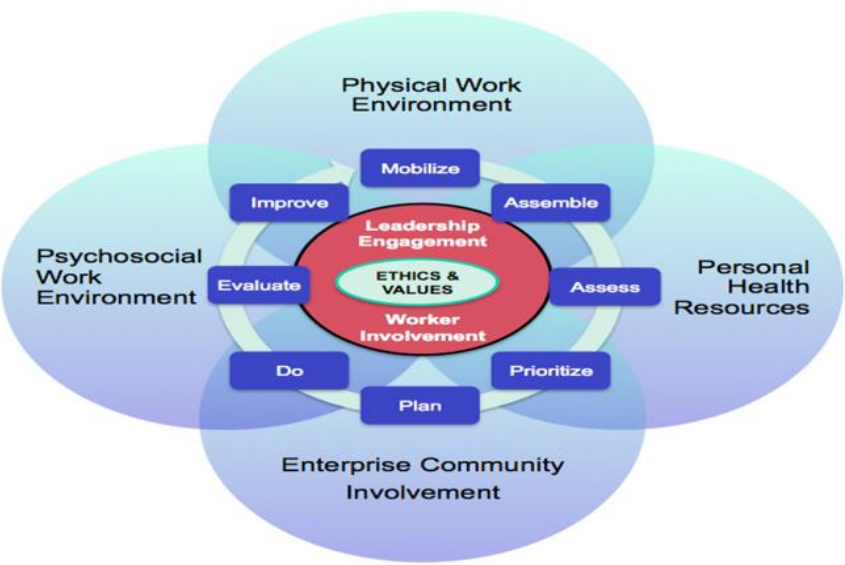

Figure 1. The WHO Model of Healthy Workplace (own figure). Adopted from Borton et al. [1] 
Unhealthy and unsafe work environment domains

1-Psychosocial

2-Physical

3-Personal health resources

4-Entreprise involvement

\begin{tabular}{|l|l|}
\hline $\begin{array}{l}\text { Care workers` health } \\
\text {-Physical } \\
\text {-Mental }\end{array}$ & $\begin{array}{l}\text { Care workers } \\
\text { outcomes } \\
\text {-Absenteeism } \\
\text { - Presenteeism }\end{array}$ \\
\hline
\end{tabular}

Figure 2. The WHO conceptual framework for business case .Adopted from Borton et al. [1] 
Table 1. Facility and care worker characteristics and psychosocial work environment factors

\begin{tabular}{ccc}
\hline Facility characteristics & n (\%) & Mean (SD) \\
\hline Language speaking region & \\
German & $123(75.9)$ \\
French & $30(18.5)$ \\
Italian & $9(5.6)$
\end{tabular}

Profit status

Public

$60(37.0)$

Private subsidized

$43(26.5)$

Private

$59(36.4)$

Nursing home size

Small (20-49beds)

$63(38.9)$

Medium (50-99 beds)

Large ( $\geq 100$ beds)

$24(14.8)$

\section{Care worker characteristics}

\section{Gender}

Male

$248(7.8)$

Female

$$
\begin{aligned}
& \text { Age groups (years) } \\
& \text { Up to } 30 \\
& 31-40 \\
& 41-50 \\
& >50
\end{aligned}
$$

Professional category

Registered Nurse

887(27.9)

Licensed practical nurse

$744(23.4)$

Certified nursing assistant

$613(19.3)$

Nurse Aide

$932(29.3)$

\section{Employment percentage}

Up to $50 \%$

$784(24.7)$

$>50 \%$

Agency Staff
Yes
No
Experience in nursing (years)
Up to 5
5 to 10
11 to 15
16 to 20
$>20$

$201(6.3)$

2975 (93.7)

$660(20.8)$

$731(23.0)$

$593(18.7)$

$412(13.0)$

$780(24.6)$ 


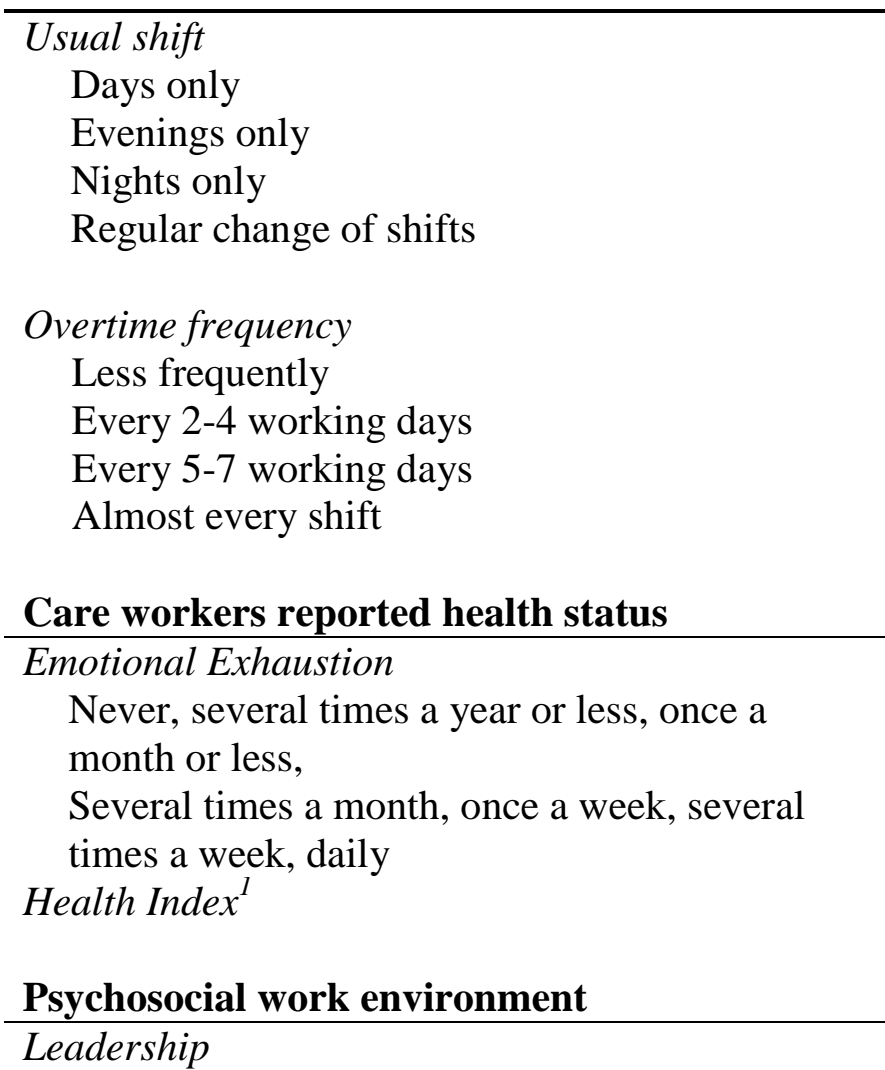

$1421(44.7)$

$198(6.2)$

$391(12.3)$

$1166(36.7)$

$2423(76.3)$

$251(7.9)$

443 (13.9)

59 (1.9)
$1978(62.3)$

$1198(37.7)$

$1.54(0.82)$

$0.90(0.66)$

$0.67(0.58)$

$3.84(0.82)$

$3049(96.0)$

Very low, rather low

Collaboration with unit supervisor

Very low, rather low

$300(9.4)$

Rather high, very high

$2876(90.6)$

Support from other personnel

Strongly disagree, slightly disagree, neutral

Slightly agree, strongly agree

Autonomy

Strongly disagree, slightly disagree

$601(18.9)$

Slightly agree, strongly agree 
Job satisfaction

Very dissatisfied, rather dissatisfied

$396(12.5)$

Rather satisfied, very satisfied

$2780(87.5)$

${ }^{1}$ Health index included self reported back pain, joint pain, tiredness, sleeplessness, and headache during the past 4 weeks prior to the survey. 
Table 2. Prevalence of absenteeism and presenteeism, n (\%)

\begin{tabular}{lcc}
\hline Care worker reported & Absenteeism & Presenteeism \\
\hline 0 days & $2713(85.4)$ & $2129(67.0)$ \\
1 to 2 days & $285(9.0)$ & $512(16.1)$ \\
$\geq 3$ days & $178(5.6)$ & $535(16.8)$ \\
Total 1 and more days & $463(14.6)$ & $1047(32.9)$ \\
\hline
\end{tabular}


Table 3. Association between work environment factors and absenteeism and presenteeism

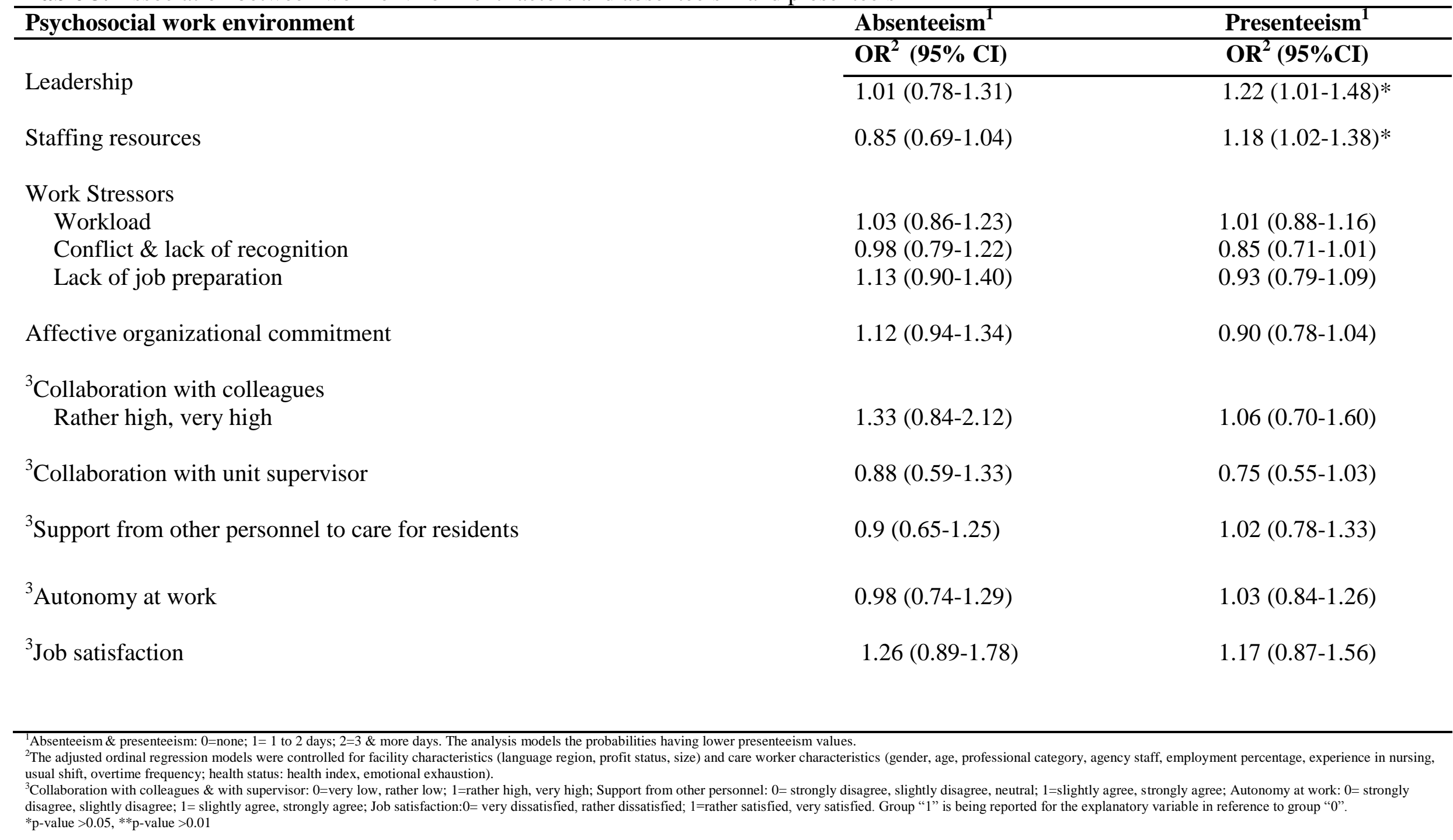


